\section{PREDICTION OF CONTRAST INDUCED NEPHROPATHY USING NOVEL BIOMARKERS FOLLOWING ELECTIVE CONTRAST CORONARY ANGIOGRAPHY}

'Michael Connolly*, Michelle Kinnin, David Mc Eneaney, lan Menown, Neal Morgan, ${ }^{2}$ Mark Harbinson. ${ }^{\prime}$ Craigavon Area Hospital; ${ }^{2}$ Queens University Belfast; *Presenting Author

\subsection{6/heartjnl-2016-309890.208}

Introduction Chronic Kidney Disease (CKD) is a risk factor for contrast induced nephropathy (CIN), defined as an increase in serum creatinine of $>25 \%$ from baseline or a delta rise of $>26.5 \mu \mathrm{mol} / \mathrm{L}$ within $48 \mathrm{~h}$. Early diagnosis of CIN requires validated novel biomarkers.

Methods A prospective observation study of 301 consecutive CKD patients undergoing elective invasive coronary angiography was performed. Low-osmolar contrast was standard. Demographics and Mehran risk score were recorded. Samples for plasma neutrophil gelatinase-associated lipocalin (NGAL), serum liver fatty acid-binding protein (L-FABP), serum kidney injury marker 1 (KIM-1), serum interleukin 18 (IL-18) and serum creatinine were taken at $0,1,2,4,6$ and $48 \mathrm{~h}$ post contrast. Urinary NGAL and urinary cystatin C (CysC) were collected at 0,6 and $48 \mathrm{~h}$. Incidence of major adverse clinical events (MACE); acute myocardial infarction, heart failure hospitalisation, stroke and death were recorded at 1 year.

Results CIN occurred in 28 (9.3\%) patients and were independently associated with older age, diabetes, higher Mehran score, larger contrast volume and anaemia $(p<0.05)$. Logistic regression analysis showed diabetes, CKD stage and GFR to be most predictive of CIN. The predictive power of plasma NGAL was greatest at $6 \mathrm{~h}$ with median levels of $1,337 \mathrm{ng} / \mathrm{ml}$ in CIN patients compared with $931 \mathrm{ng} / \mathrm{ml}$ in non-CIN patients ( $\mathrm{p}=0.002$, AUC 0.71 , sensitivity $75.0 \%$, specificity 96.1\%, OR 2.86), see figure 1 and table 1. L-FABP performed best at $4 \mathrm{~h}$ with median levels of $10.7 \mathrm{ng} / \mathrm{ml}$ in CIN patients compared with $6.2 \mathrm{ng} / \mathrm{ml}$ in non-CIN patients, $\mathrm{p}=0.001$, AUC 0.69 , sensitivity $42.3 \%$, specificity $90.2 \%$, OR 6.75 , Figure 1 and Table 1 . Median urinary NGAL was higher only after $48 \mathrm{~h}, 487 \mathrm{ng} / \mathrm{ml}$ in CIN patients versus $155 \mathrm{ng} / \mathrm{ml}$ in non-CIN patients, $\mathrm{p}=0.008$, AUC 0.63. CysC, IL-18 and KIM-1 were not predictive at any time-point $(p>0.05)$. A Mehran score $\hat{\mathrm{a}} \%$ ¥ 10 performed prior to procedure achieved an AUC of $0.65, p=0.006$. MACE occurred in $7(25.0 \%)$

Abstract 208 Table 1 Summary of NGAL (ng/ml) and L-FABP (ng/ $\mathrm{ml}$ ) in AKI and non-AKI patients

\begin{tabular}{|c|c|c|c|c|c|}
\hline Biomarker & $\begin{array}{c}\text { Time } \\
\text { (hr) }\end{array}$ & $\begin{array}{c}\text { Median } \\
\text { CIN }\end{array}$ & $\begin{array}{c}\text { Median } \\
\text { non-CIN }\end{array}$ & AUC & $\begin{array}{c}\text { P } \\
\text { value }\end{array}$ \\
\hline \multirow{4}{*}{ NGAL } & 0 & 1108 & 959 & 0.62 & 0.046 \\
\cline { 2 - 6 } & 2 & 1219 & 883 & 0.68 & 0.004 \\
\cline { 2 - 6 } & 4 & 1224 & 902 & 0.65 & 0.014 \\
\cline { 2 - 6 } & 6 & 1337 & 931 & 0.71 & 0.002 \\
\hline \multirow{4}{*}{ L-FABP } & 0 & 11.3 & 7.3 & 0.65 & 0.011 \\
\cline { 2 - 6 } & 2 & 11.3 & 6.9 & 0.67 & 0.007 \\
\cline { 2 - 6 } & 4 & 10.7 & 6.2 & 0.69 & 0.001 \\
\cline { 2 - 6 } & 6 & 8.3 & 6.7 & 0.63 & 0.045 \\
\hline
\end{tabular}

Sensit: sensitivity; specif: specificity; PPV: positive predictive value; NPV: negative predictive value; RR: relative risk; OR: odds ratio
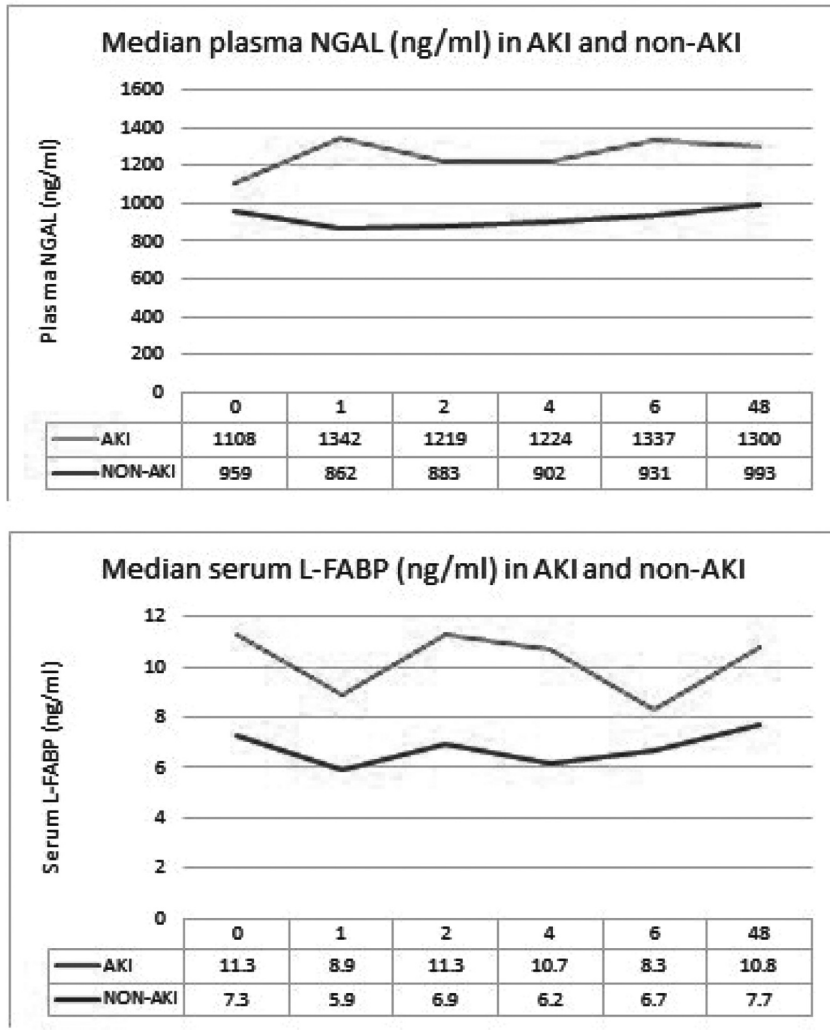

Abstract 208 Figure 1 Median plasma NGAL (ng/ml) and serum LFABP $(n g / m l)$ in AKI and non-AKI

CIN patients but only 17 (6.2\%) non-CIN patients $(\mathrm{p}<$ 0.001). CIN cases also had considerably higher mortality $(10.7 \%$ compared to $3.3 \%, p=0.037)$. Exploratory analysis showed that the combination of Mehran score $>10,6 \mathrm{hr}$ NGAL and $4 \mathrm{hr}$ L-FABP improved specificity to $96.7 \%$. Figure 2 highlights a proposed pathway of how biomarkers could be used to identify CIN early and facilitate timely therapeutic intervention to reduce morbidity and mortality.

Conclusions/implications Mehran risk score, $6 \mathrm{~h}$ plasma NGAL and $4 \mathrm{~h}$ serum L-FABP performed best at early CIN prediction. CIN patients were four times more likely to develop MACE and had a trebling of mortality risk at 1 year. The implications of our results, translated to the design of safer elective coronary intervention services able to more efficiently manage the increasing volume of contrast studies, should be a key health priority for cardiac and renal services.

\section{WHOLE EXOME SEQUENCING IDENTIFIES GENETIC CAUSE OF HISTIOCYTOID CARDIOMYOPATHY}

${ }^{1}$ Gillian Rea*, ${ }^{2}$ Tessa Homfray, ${ }^{2}$ Jan Till, ${ }^{2}$ Ferran Roses-Noguer, 'Rachel J Buchan, ${ }^{1}$ Sam Wilkinson, ${ }^{1}$ Roddy Walsh, ${ }^{3}$ Shane McKee, ${ }^{3}$ Fiona I Stewart, ${ }^{4}$ Victoria Murday, ${ }^{5}$ Robert W Taylor, ${ }^{2}$ A John Baksi, ${ }^{2}$ Sanjay K Prasad, ${ }^{1}$ Paul JR Barton, ${ }^{1}$ James S Ware, ${ }^{7}$ Stuart A Cook. 'Imperial College London \& Royal Brompton and Harefield NHS Foundation Trust; ${ }^{2}$ Royal Brompton \& Harefield NHS Foundation Trust; ${ }^{3}$ Northern Ireland Regional Genetics Service; ${ }^{4}$ Department of Clinical Genetics, Laboratory Medicine, The Queen Elizabeth University Hospital; ${ }^{5}$ Wellcome Trust Centre for Mitochondrial Research; ${ }^{6}$ National Heart Centre; *Presenting Author

10.1136/heartjnl-2016-309890.209 
Current Pathway

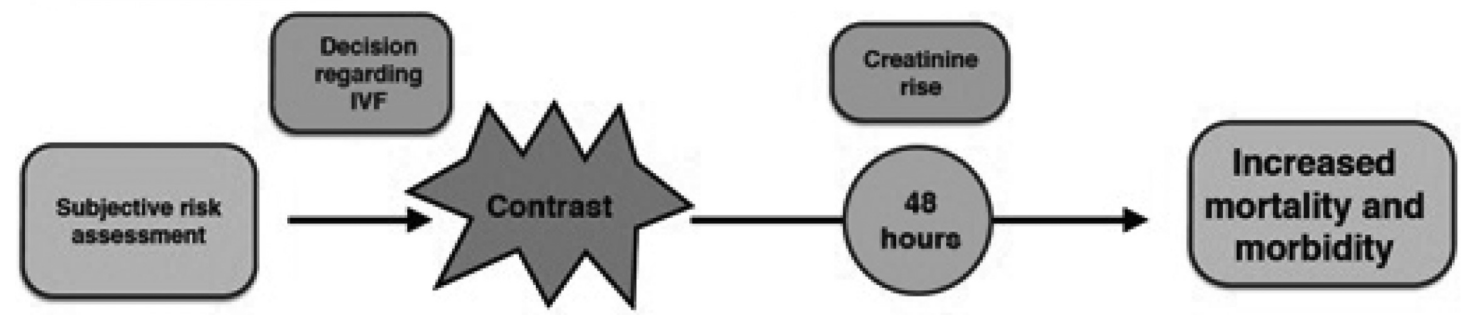

Proposed Pathway

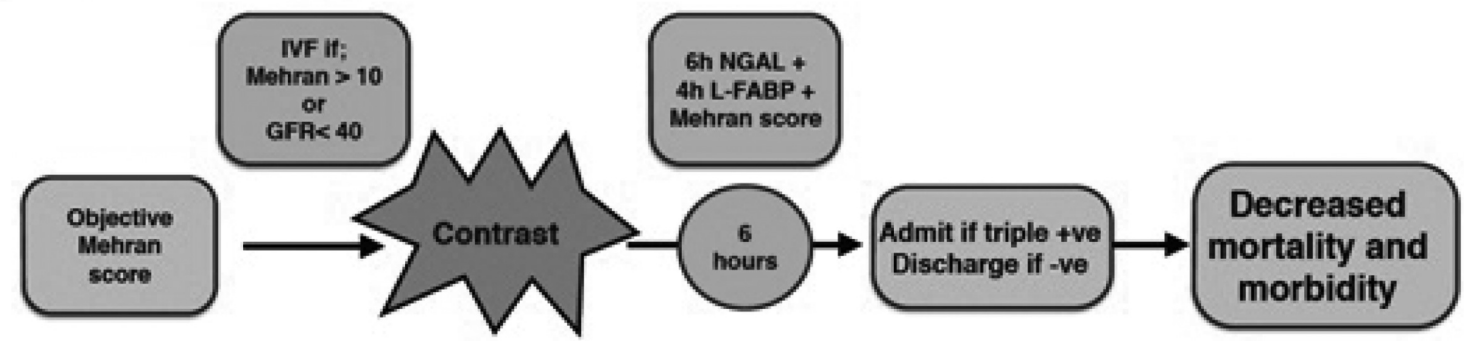

Abstract 208 Figure 2 Proposed patient pathways

Histiocytoid cardiomyopathy (CM) is a rare, distinctive form of cardiomyopathy, characterised by malignant arrhythmias and associated sudden death. $\sim 90 \%$ of cases present in females $<2$ years of age. We undertook whole exome sequencing (WES) in five unrelated affected females, including one with parental samples available. In the family trio we identified a de novo nonsense mutation in NDUFB11 (c.262C>T; p. Arg88*), located on the $\mathrm{X}$ chromosome and encoding a component of the mitochondrial respiratory chain (MRC). Mutations in NDUFB11, including one identical to the one we describe here, have been reported to cause microphthalmia and linear skin defects syndrome (MLS). During the course of our studies, additional mutations in NDUFB11 were associated with Histiocytoid CM by another group. Four of the affected individuals in our study did not carry variants in NDUFB11. Heterozygous mutations in HCCS (which encodes an important mitochondrially-targeted protein) and $C O X 7 B$ (which, like NDUFB11, encodes a protein of the MRC) have also previously been identified in MLS syndrome including a case with features of both MLS syndrome and Histiocytoid CM. However, a systematic review of WES data from previously published cases, alongside the four additional cases presented here, did not identify any further mutations in these genes in Histiocytoid CM. We conclude thattruncating variants in NDUFB11 link the distinct phenotypes of Histiocytoid CM and MLS syndrome. Screening for malignant arrhythmias and cardiomyopathy would be appropriate in individuals with MLS syndrome. Additional nuclear encoded mitochondrial or mitochondrial DNA genes are good candidates for further causes of both Histiocytoid CM and MLS syndrome.

\section{CLINICAL UTILITY OF GENE PANEL AND CLINICAL EXOME TESTING IN CARDIAC DISEASE}

${ }^{1}$ Mary Gable*, 'Chris Buxton, 'Geoff Woodward, 'Marc Wadsley, 'Julie Honeychurch, ${ }^{1}$ Davies Joanne, ${ }^{2}$ Ruth Newbury-Ecob, ${ }^{2}$ Karen Low, ${ }^{2}$ Alan Donaldson, ${ }^{2}$ Josphine Affleck, ${ }^{1}$ Rebecca Whittington, ${ }^{1}$ Mark Greenslade, 'Maggie Williams. 'Bristol Genetics Laboratory; ${ }^{2}$ Department of Clinical Genetics; *Presenting Author

\subsection{6/heartjnl-2016-309890.210}

Cardiac disease is genetically heterogeneous with genes associated with multiple cardiac diseases, multiple causal genes per disease, and often multiple variants in one or more genes contributing to disease presentation. Gene panel testing, either through a specific targeted design, or by virtual analysis from the exome/clinical exome is an ideal approach for genetic diagnosis and provides information regarding complexity of these diseases.

Bristol Genetics Laboratory provides a targeted gene panel for paediatric cardiomyopathy (PC) (71 genes, Agilent SureSelect) and a variety of virtual gene panels from Agilent Focussed Exome; Congenital Heart Defect (CHD) (38 genes), Aortopathy (28 genes), Arrhythmia (54 genes), Cardiomyopathy (119 genes), Connective Tissue (42 genes) Molecular Autopsy (208 genes) and bicuspid aortic valve (8 genes). Whole clinical exome analysis (6110 genes) with phenotypic prioritisation of variants based on HPO terminology using Exomiser can also be performed for patients with complex phenotypes whose phenotype does not clearly fit into a predefined gene panel or for patients who tested negative for a specific panel. 